\title{
Structural and thermoelectric properties of Zintl-phase CaLiPn (Pn: As, Sb, Bi)
}

\author{
ANOOP K CHANDRAN
}

\author{
A Thesis Submitted to \\ Indian Institute of Technology Hyderabad \\ In Partial Fulfillment of the Requirements for \\ The Degree of Master of Physics
}

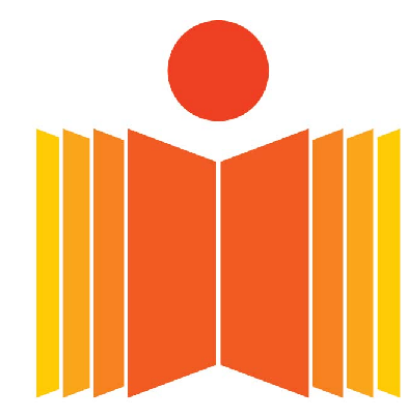

भारतीय प्रौद्योगिकी संस्थान हैदराबाद

Indian Institute of Technology Hyderabad

Department of Physics

April 2016 


\section{Declaration}

I declare that this written submission represents my ideas in my own words, and where ideas or words of others have been included, I have adequately cited and referenced the original sources. I also declare that I have adhered to all principles of academic honesty and integrity and have not misrepresented or fabricated or falsified any idea/data/fact/source in my submission. I understand that any violation of the above will be a cause for disciplinary action by the Institute and can also evoke penal action from the sources that have thus not been properly cited, or from also permission has not been taken when needed.

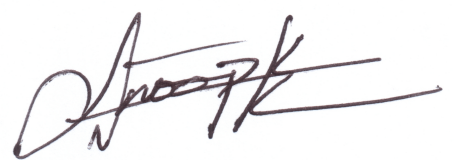

(ANOOP K CHANDRAN)

PH14MSCST11003 


\section{Approval Sheet}

This thesis entitled "Structural and thermoelectric properties of Zintl-phase CaLiPn (Pn: As, $\mathrm{Sb}, \mathrm{Bi})$ " by ANOOP K CHANDRAN is approved for the degree of Master of Physics from IIT Hyderabad.

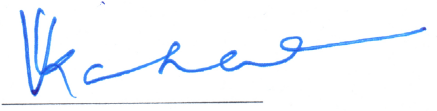

Dr. V KANCHANA (Adviser)

Dept. of Physics

IITH

\section{R. Bhuvanarh}

(Examiner)

Dept. of Physics

IITH

Vandare Shenme

(Examiner)

Dept. of Physics

IITH

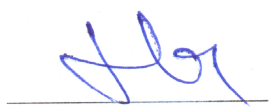

(Examiner)

Dept. of Physics

IITH

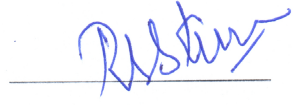

(Examiner)

Dept. of Physics

IITH

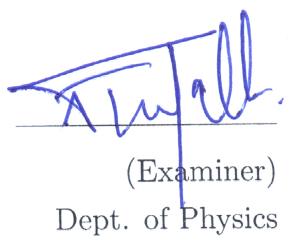

IITH 


\section{Acknowledgements}

I would like to express my sincere gratitude to my advisor, Dr V. Kanchana for the continuous support of my project work. Her motivation and constructive criticism helped me to complete the work. From finding out my talent to putting my skills to right areas of the work she guided me with patience and enthusiasm and helping me in all the time of my project and writing of this thesis.

During the time of my project, I have learned a lot of things about computational programming and about an approach to research in general from Vijay Kumar Gudelli, Sreeparvathy P C, Shwetha Gummula and P V Sreenivasa Reddy, who are the research scholars under my supervisor. I could not have imagined having a better team and mentors for my project study.

I would also like to extend my gratitude towards the head of the department Prof. Anjan Kumar Giri and all faculty members of physics department for their valuable lectures.

I am also grateful to my friends for their immense support and encouragement. 


\begin{abstract}
First-principles calculations were carried out to study the structural, mechanical, dynamical and transport properties of zintl phase materials $\mathrm{CaLi} P n(P n=\mathrm{As}, \mathrm{Sb}$ and $\mathrm{Bi})$. We have used two distinct density functional approaches to investigate these properties. The plane wave pseudopotential approach was used to study the structural and dynamical properties. The full potential linear augment plane wave method has been used to study the electronic structure, mechanical and thermoelectric properties. The calculated ground-state properties agree quite well with experimental values. The calculated electronic structure shows the investigated compounds to be direct band gap semiconductors. Further we have calculated the thermoelectric properties of all the investigated compounds as a function of both carriers at various temperatures. We found a high thermopower for both the concentrations, especially with n-type doping to be more favourable, which enabled us to predict that $\mathrm{CaLi} P n$ might have promising applications as a good thermoelectric material. Further the phonon dispersion curves of the investigated compounds showed flat nature of the phonon bands and we also find low lying optical and acoustic modes are cutting each other at the lower frequency range, which indicate that the investigated compounds might have an reasonable low thermal conductivity.
\end{abstract}




\section{Contents}

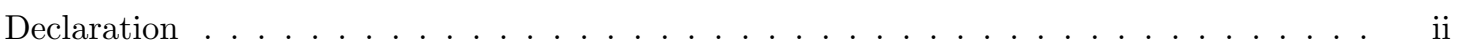

Acknowledgements ........................ iv

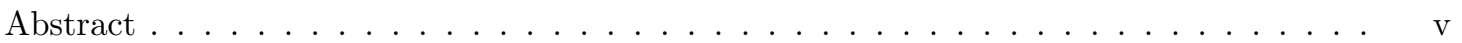

1 Introduction $\quad 1$

2 Theoretical Background $\quad 3$

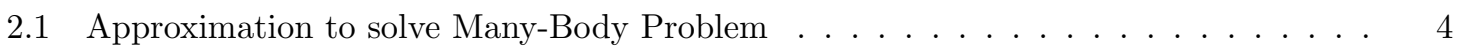

2.1.1 Born-Oppenheimer Approximation . . . . . . . . . . . . . . . 4

2.1.2 The Hartree approximation $\ldots \ldots \ldots \ldots$

2.1 .3 Hartree-Fock Method . . . . . . . . . . . . . . . . . . . . 5

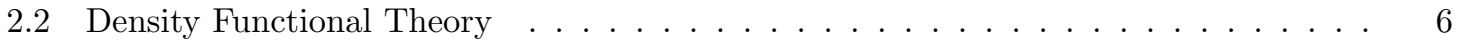

2.2 .1 Thomas-Fermi Equation . . . . . . . . . . . . . . . . 6

2.2.2 Hohenberg - Kohn Theorem . . . . . . . . . . . . . . . . . 7

2.2 .3 Khon-Sham Method . . . . . . . . . . . . . . . . . 7

2.2 .4 Local Density Approximation $(\mathrm{LDA}) \ldots \ldots \ldots \ldots$

2.2.5 Generalised Gradient Approximation (GGA) $\ldots \ldots \ldots$

2.2.6 Tran-Blaha modified Becke-Johnson potential (TB-mBJ) . . . . . . . . . 10

2.3 Introduction to Thermoelectric Materials _ . . . . . . . . . . . . . . . 10

3 Methodology 13

4 Results and Discussions

$4.1 \quad$ Structural and Mechanical properties . . . . . . . . . . . . . . . . . . . . 15

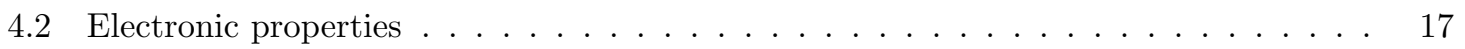

4.3 Thermoelectric properties . . . . . . . . . . . . . . . . . . . . 19

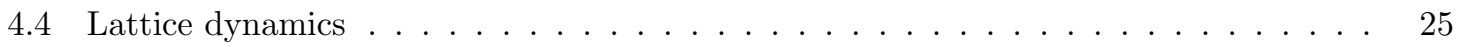


5 Conclusion

References 


\section{Chapter 1}

\section{Introduction}

Thermoelectric (TE) materials are of current interest for a number of energy related applications such as waste heat recovery, terrestrial cooling and thermoelectric power generation[1,2]. Since TE materials have the property to convert thermal energy directly to electrical energy, improvement of their efficiency[3] can lead to wider applications in energy technology. The energy conversion efficiency of a TE material is characterised by its dimensionless quantity called figure of merit, $Z T=\sigma S^{2} T / \kappa$, where $\sigma, S, T, \kappa$ are electrical conductivity, the Seebeck coefficient, the absolute temperature, and the thermal conductivity, respectively. Thermal conductivity has both electronic $\kappa_{e}$, and lattice $\kappa_{l}$, contributions, i.e. $\kappa=\kappa_{e}+\kappa_{l}$. The contradicting nature of thermopower, $|S|$, and electrical conductivity, $\sigma$, would suggest that achieving high figure of merit will require a fine tuning configuration of contributing parameters[4]. In addition to high temperature and high effective mass $m^{*}$, the carrier concentration can also ensure high thermopower. Low carrier concentration insulators and semiconductors can deliver high thermopower. However lowering carrier concentration can also result in lower electrical conductivity (since $\sigma=n e \mu$, where $n$ is the carrier concentration, $e$ is the charge of an electron and $\mu$ is the mobility). Even though metals offer high electrical conductivity which translate to high carrier concentration, they may not be suitable as a high performing TE material because not only the carrier concentration but also the mobility, which depend on effective mass is also in disagreement with that of high thermopower. Because of this conflicting nature, a compromise has to be made between high mobility and high effective mass. Typically materials with small electro negativity differences posses high mobility and low effective mass and materials with narrow bands can offer low mobility and high effective mass[5] because of the relationship between density of states and the whole dispersion relation in the momentum space. In addition to that an 
other conflict in parameters stem from acquiring low thermal conductivity. The difficulty of achieving low electronic thermal conductivity because of its proportionality to electrical conductivity according to Wiedmann-Franz law and because of this at a fixed temperature electronic thermal conductivity cannot be lowered without compromising $Z T$. Nevertheless lattice thermal conductivity can be reduced, thereby reducing the thermal conductivity. In effect an efficient TE material must have a low lattice thermal conductivity as that of an amorphous glass. Zintl phase compounds have complex structures which enable them to have low thermal conductivity $[6,7,8]$.

Research over the years point to the direction that zintl phase materials offer desired characteristics of a good thermoelectric material. These materials are considered to be valence precise[9] and majority of them form the requisite small band gap semiconductors with complex structures. Materials of this class often contains cationic sites that allow for the addition of disordered scattering and the change in carrier concentration, which results in tuning of electronic properties[13]. This characteristic of zintl materials allow control of the carrier concentration through precise doping without disrupting the carrier mobility. Since $Z T$ is sensitive to carrier concentration, this tune-ability attribute of zintl phases can be used to improve the figure of merit. Over the years we have known materials with $Z T>1$ especially from zintl phases[14] and they offer favourable thermal conductivity at temperatures near $1200 \mathrm{~K}$ because of their complex crystal structures and optimal charge-transport because of their electronic structure[15]. $C a_{5} A l_{2} S b_{6}, Y b_{14} A l S b_{11}$, and $\mathrm{Sr}_{3} A l S b_{3}[6,7,8]$ are a few examples of such compounds. $C a_{5} A l_{2} S b_{6}$ has a low lattice thermal conductivity $(0.6 \mathrm{~W} / \mathrm{mK}$ at $850 \mathrm{~K})$ and a ZT which is more than 0.6 at $1000 \mathrm{~K}$. An optimally doped $S r_{3} A l S b_{3}$ and $Y b_{14} A l S b_{11}$ can achieve ZT of 1.0 at high temperatures such as 1000 to $1250 \mathrm{~K}$, and they have lattice thermal conductivity less than $0.75 \mathrm{~W} / \mathrm{mK}$ at 1000 - $1300 \mathrm{~K}$ respectively. Recently studied strontium based pnictogen compound SrLiAs has been demonstrated to have characteristics of a reasonable TE material[17]. This provoke us to search for other possible new zintl phase materials for a better TE candidates. In the present study, we are interested in investigating the calcium based zintl phase materials of $\mathrm{CaLiPn}(P n=\mathrm{As}, \mathrm{Sb}$, and $\mathrm{Bi})$ for a possible good TE properties.

The present work is a theoretical study of $\mathrm{CaLiPn}(P n=\mathrm{As}, \mathrm{Sb}, \mathrm{Bi})$ to determine its $\mathrm{TE}$ properties. This thesis is organized as follows: chapter 2 describes the theoretical background, and chapter 3 explains computational aspects of the present study. In chapter 4 we have presented the results and discussions, and finally chapter 5 gives the conclusions and future work. 


\section{Chapter 2}

\section{Theoretical Background}

The fundamental postulates of quantum mechanics assert that microscopic system are described by wave function that completely characterize all the physical properties of the system called observables of various operators defined in quantum mechanics. For a solid system with large number of such microscopic systems (atoms) and having a huge number of particles, it is difficult to solve the Schrödinger equation $H \psi=E \psi$. For such systems the main interest is to find approximate solution of non-relativistic time independent Schrödinger equation. In general Hamiltonian of such a system is defined by kinetic energy of the electron and nuclei, electron-electron interaction, nucleinuclei interaction, electron-nuclei interaction. These interactions can be expressed in the following Hamiltonian (in atomic units $\hbar=|e|=m_{e}=\frac{1}{4 \pi \epsilon_{0}}=1, E=27.21 \mathrm{eV}, r=a_{0}$ ),

$$
H=-\sum_{i} \frac{1}{2} \nabla_{i}^{2}-\sum_{I} \frac{1}{2} \nabla_{I}^{2}+\sum_{\substack{I, J \\ I \neq J}} \frac{1}{2} \frac{1}{\left|\overrightarrow{r_{i}}-\overrightarrow{r_{j}}\right|}-\sum_{i, I} \frac{Z_{I}}{\left|\overrightarrow{R_{I}}-\overrightarrow{r_{i}}\right|}+\sum_{\substack{I, J \\ I \neq J}} \frac{Z_{I} Z_{J}}{\left|\overrightarrow{R_{I}}-\overrightarrow{R_{J}}\right|}
$$

The small indexes(i,j) are referring to electrons and capital $(\mathrm{I}, \mathrm{J})$ are for nuclei. The $H$ of the above equation is known as many body Hamiltonian. Approximations are needed to solve this many body Hamiltonian, which are explained in detail below. 


\subsection{Approximation to solve Many-Body Problem}

\subsubsection{Born-Oppenheimer Approximation}

Since nuclei are much heavier than electrons, their velocities are much smaller. To a good approximation, the Schrödinger equation can be separated into two parts: One part describes the electronic wavefunction for a fixed nuclear geometry. The second describes the nuclear wavefunction, where the electronic energy plays the role of a potential energy. In other words, the kinetic energy of the nuclei can be treated separately. This is the Born-Oppenheimer (BO) approximation. As a result, the electronic wave function depends only on the positions of the electrons. We assume that electrons move in an electrostatic field generated by the nuclei. Since we consider the nuclei to be at rest we can rewrite the Hamiltonian as

$$
H=-\sum_{i} \frac{1}{2} \nabla_{i}^{2}-\sum_{\lambda} \frac{1}{2} \nabla_{I}^{2}+\sum_{\substack{i, j \\ i \neq j}} \frac{1}{2} \frac{1}{\left|\overrightarrow{r_{i}}-\overrightarrow{r_{j}}\right|}-\sum_{i, I} \frac{Z_{I}}{\left|\overrightarrow{R_{I}}-\overrightarrow{r_{i}}\right|}+\sum_{\substack{I, J \\ I \neq J}} \frac{Z_{I} Z_{J}}{\left|\overrightarrow{R_{I}}-\overrightarrow{R_{J}}\right|}
$$

In $\mathrm{BO}$ approximation, the total wave function is limited to one electronic surface, i.e. a particular electronic state. The BO approximation is usually very good, but breaks down when two (or more) electronic states are close in energy at particular nuclear geometries. Further approximations are needed to solve this Hamiltonian.

\subsubsection{The Hartree approximation}

For non interacting particles we can write the wave function as a product of individual particle wave function. Hartree considered each system to obey a Schrödinger equation and one can write the wave equation for an $n$ particle system as,

$$
\psi\left(\overrightarrow{r_{i}}\right)=C_{N} \prod_{i}^{n} \phi_{i}\left(\overrightarrow{r_{i}}\right)
$$

Here interaction of one electron with the others are incorporated in an average way. We can write the Schrödinger equation as

$$
\left(\frac{1}{2} \nabla^{2}+V_{\text {ext }}(\vec{r})+V_{\text {additional }}(\vec{r})\right) \phi_{i}=\epsilon_{i} \phi_{i}
$$


Where

$$
V_{e x t}(\vec{r})=-\sum_{i, I} \frac{Z_{I}}{\left|\overrightarrow{R_{I}}-\overrightarrow{r_{i}}\right|}+\sum_{\substack{I, J \\ I \neq J}} \frac{Z_{I} Z_{J}}{\left|\overrightarrow{R_{I}}-\overrightarrow{R_{J}}\right|}
$$

Which is the last two terms from eq-(1) and

$$
V_{\text {additional }}(\vec{r})=\frac{1}{2} \sum_{\substack{i, j \\ i \neq j}} \frac{1}{\left|\overrightarrow{r_{i}}-\overrightarrow{r_{j}}\right|}
$$

$V_{\text {additional }}$ is the Coulomb interaction. The limitations of this approximation are, they do not include correlation, the wavefunction is not antisymmetric and it does not remove the $(n, l)$ - accidental degeneracy of the hydrogenoid atom.

\subsubsection{Hartree-Fock Method}

One must include the antisymmetry as well in order to describe a wave function. The slater determinant takes care of the spin. Interchanging the position of two electrons is equivalent to interchanging the corresponding column. If two electrons at the same spin interchange positions, $\psi^{D}$ must change sign. This is known as exchange property and is the manipulation of Pauli principle. The wave function approach to systems with many atom does not offer a satisfactory approach to the one electron approximation. One of the most common ways of dealing with many - Fermion problem is to assume that each electron can be considered separately in the one electron approximation.

$$
\begin{gathered}
\psi^{D}(\vec{r})=\frac{1}{\sqrt{N !}}\left|\begin{array}{cccc}
\chi_{1}\left(\overrightarrow{r_{1}}\right) & \chi_{2}\left(\overrightarrow{r_{1}}\right) & \ldots & \chi_{n}\left(\overrightarrow{r_{1}}\right) \\
\chi_{1}\left(\overrightarrow{r_{2}}\right) & \chi_{2}\left(\overrightarrow{r_{2}}\right) & \ldots & \chi_{n}\left(\overrightarrow{r_{2}}\right) \\
\vdots & \vdots & \ddots & \vdots \\
\chi_{1}\left(\overrightarrow{r_{n}}\right) & \chi_{2}\left(\overrightarrow{r_{n}}\right) & \ldots & \chi_{n}\left(\overrightarrow{r_{n}}\right)
\end{array}\right| \\
H=\left(-\sum_{i} \frac{1}{2} \nabla_{i}^{2}+V_{\text {ext }}\left(\overrightarrow{r_{i}}\right)\right)+\frac{1}{2} \sum_{\substack{i, j \\
i \neq j}} \frac{1}{\left|\overrightarrow{r_{i}}-\overrightarrow{r_{j}}\right|}
\end{gathered}
$$

Solving the Schrödinger equation with the slater determinant as the wave function we will arrive at the equation given below,

$$
\left(\frac{-1}{2} \nabla^{2}+V_{e x t}+\int \frac{\rho\left(\overrightarrow{r^{\prime}}\right)}{\left|\vec{r}-\overrightarrow{r^{\prime}}\right|} d r^{\prime}-\frac{1}{2} \sum_{i, j, \sigma} \int \frac{\phi_{j, \sigma}^{*}\left(\overrightarrow{r^{\prime}}\right) \phi_{i, \sigma}\left(\overrightarrow{r^{\prime}}\right) \phi_{j, \sigma}(\vec{r})}{\phi_{i, \sigma}\left(\overrightarrow{r^{\prime}}\right)\left|\vec{r}-\overrightarrow{r^{\prime}}\right|} d r d r^{\prime}\right) \phi_{i, \sigma}(\vec{r})=\epsilon_{i} \phi_{i, \sigma}(\vec{r})
$$


This is called the Hartree-Fock equation. The Hartree-Fock equations describe non-interacting electrons under the influence of a mean field potential consisting of the classical Coulomb potential and a non-local exchange potential. The correlation energy is the difference between the full ground state energy and the exchange correlation energy. The correlation energy accounts for the energy lowering due to quantum fluctuations. Since this is still a $3 \mathrm{~N}$ dimensional problem and solving this would be difficult. In addition to that regardless of this method's success in describing various systems, it failed because of the poor exchange and correlation limits of electrons. Adopting further approximations were needed to minimise the problem. The triumph came with the formulation of Density Functional Theory in solving this complex problem. The same is explained in the following sections.

\subsection{Density Functional Theory}

Density Functional Theory (DFT) emanate from Hohenberg-Kohn theory and Kohn-Sham equation. This uses density as a fundamental quantity instead of wavefunction, which leads to a situation where the complexity of the problem can be effectively reduced from $3 \mathrm{~N}$ to 3 . The density of the electron can be $\rho(r)$ and can be expressed as,

$$
\rho(r)=N \int d^{3} r_{2} d^{3} \ldots d^{3} r_{N} \psi\left(r, r_{2}, \ldots r_{N}\right) \psi^{*}\left(r, r 2 \ldots r_{N}\right)
$$

The indication that the density can be used as a fundamental parameter as the wavefunction is originated from Thomas-Fermi equation, which is explained in the following section.

\subsubsection{Thomas-Fermi Equation}

Thomas and Fermi independently considered the first three terms of the Hartree-Fock equation. At that time they were not aware of the exchange energy and neglected the correlation term. For a plane wave systems like homogeneous electron gas one can solve the HF equation and find out the approximate energy as follows. For a homogeneous electron gas $\phi_{i}(\vec{r})=\frac{1}{\sqrt{V}} e^{i \vec{k} \cdot \vec{r}}$. If we use this and minimise the total energy we get,

$$
\frac{5 C_{k}}{3} \rho(\vec{r})^{2 / 3}+V_{\text {ext }}+\frac{1}{2} \int \frac{\rho\left(\overrightarrow{r^{\prime}}\right)}{\left|\vec{r}-\overrightarrow{r^{\prime}}\right|} d r^{\prime}-\left(\frac{3 \rho(\vec{r}}{\pi}\right)^{1 / 3}=\mu
$$


The Lagrangian multiplier $\mu$ will be the chemical potential of the homogeneous electron gas. It shows that given a density it will give a number. The idea of using density as a fundamental variable is originated from this concept. Thomas-Fermi becomes relevant when the system is dense or the kinetic energy is more.

\subsubsection{Hohenberg - Kohn Theorem}

The question of treating $\rho(\vec{r})$ as a fundamental variable is answered by Hohenberg - Khon Theorem (HK). In 1964 Hohenburg and Kohn proved the two theorems. The first theorem may be stated as follows: for any system of interacting particles in an external potential $V_{\text {ext }}(\vec{r})$, the density is uniquely determined. If this statement is true then it immediately follows that the electron density uniquely determines the Hamiltonian operator. The second theorem establishes a variational principle: For any positive definite trial density $\rho_{i}(\vec{r})$, such that $\int \rho_{i}(\vec{r}) d r=N$ then $E\left[\rho_{i}(\vec{r})\right] \geq E_{o}$

HK theorem provides evidence for the one-to-one correspondence between external potential $V_{\text {ext }}(\vec{r})$ and ground state density $\rho_{0}(r)$. It gives good approximation to the ground density as well as the energy. But still we have to solve many electron shrodinger equation. A practical implementation can be carried out using Kohn-Sham method which is discussed in the next section.

\subsubsection{Khon-Sham Method}

Since density is a fundamental variable we can re write the Shrodinger equation eq-(2.11) as,

$$
E[\rho(\vec{r})]=T[\rho(\vec{r})]+V_{e x t}[\rho(\vec{r})]+V_{e e}[\rho(\vec{r})]
$$

Here $V_{\text {ext }}[\rho(\vec{r})]=\int V_{\text {ext }} \rho(\vec{r}) d r$. But the kinetic and electron-electron functionals are unknown. If good approximations to these functionals could be found direct minimisation of the energy would be possible. Kohn and Sham proposed the following approach to approximating the kinetic and electron-electron functionals. They introduced a fictitious system of $\mathrm{N}$ non- interacting electrons to be described by a single determinant wavefunction in $\mathrm{N}$ orbitals $\phi_{i}$. In this system the kinetic energy and electron density are known exactly from the orbitals;

$$
T_{s}[\rho(\vec{r})]=-\frac{1}{2} \sum_{i}^{N}\left\langle\phi_{i}\left|\nabla^{2}\right| \phi_{i}\right\rangle
$$

Here the suffix (s) emphasises that this is not the true kinetic energy but is that of a system of non-interacting electrons, which reproduce the true ground state density; $\rho(\vec{r})=\sum_{i}^{N}\left|\phi_{i}^{2}\right|$. The 
construction of the density explicitly from a set of orbitals ensures that it is legal and it can be constructed from an asymmetric wavefunction. The energy functional can be rearranged as;

$$
E[\rho(\vec{r})]=T_{s}[\rho(\vec{r})]+V_{e x t}[\rho(\vec{r})]+V_{H}[\rho(\vec{r})]+E_{X C}[\rho(\vec{r})]
$$

Where, $V_{H}=\frac{1}{2} \int \frac{\rho(\vec{r}) n\left(\overrightarrow{r^{\prime}}\right)}{\left|\vec{r}-\overrightarrow{r^{\prime}}\right|} d r d r^{\prime}$ and $E_{X C}$ is called exchange correlation functional;

$$
E_{X C}[\rho(\vec{r})]=T[\rho(\vec{r})]-T_{s}[\rho(\vec{r})]+V_{e e}[\rho(\vec{r})]-V_{H}[\rho(\vec{r})]
$$

which is simply the sum of the error made in using a non-interacting kinetic energy and the error made in treating the electron-electron interaction classically. This set of non-linear equations (the Kohn-Sham equations) describes the behaviour of non-interacting electrons in an effective local potential. For the exact functional, and thus exact local potential, the orbitals yield the exact ground state density. These Kohn-Sham equations have the same structure as the Hartree-Fock equations with the non-local exchange potential replaced by the local exchange-correlation potential $V_{X C}=\frac{\delta E_{X C}(\vec{r})}{\delta \rho(\vec{r})}$.

\section{Proceeding Numerically}

Since the ground state electron density is the fundamental variable, it gives us an advantage by reducing the $3 \mathrm{~N}$ dimensional problem to a 3 dimensional problem. We start with an initial guess of $\rho_{o}(\vec{r})$ then use it to calculate $V_{K S}[\rho(\vec{r})]$ which is the effective potential. Then solving the Kohn-Sham equations must be trivial task. We calculate the energy density again and obtain $\rho(\vec{r})$. And check for self consistency i.e. whether $\rho(\vec{r})$ is nearly equal to $\rho_{o}(\vec{r})$ or not. If not we proceed to do the calculation again using $\rho(\vec{r})$ as our new input ground state density and do the process again till the self consistency is acquired. If the result is self consistent, we go on to calculating energy and force etc. The self-consistent algorithm is shown in Fig. 2.1

\subsubsection{Local Density Approximation (LDA)}

LDA approximation is a key contribution by Kohn-Sham, which for computations of the quantum ground state of many-particle systems proved to be superior to both Thomas-Fermi and HartreeFock theories, the basis of the local density approximation for the exchange and correlation energy functional is the theory of the homogeneous electron gas. This is a most important model system, which of course does not exist in nature, but which can nowadays theoretically be treated with 


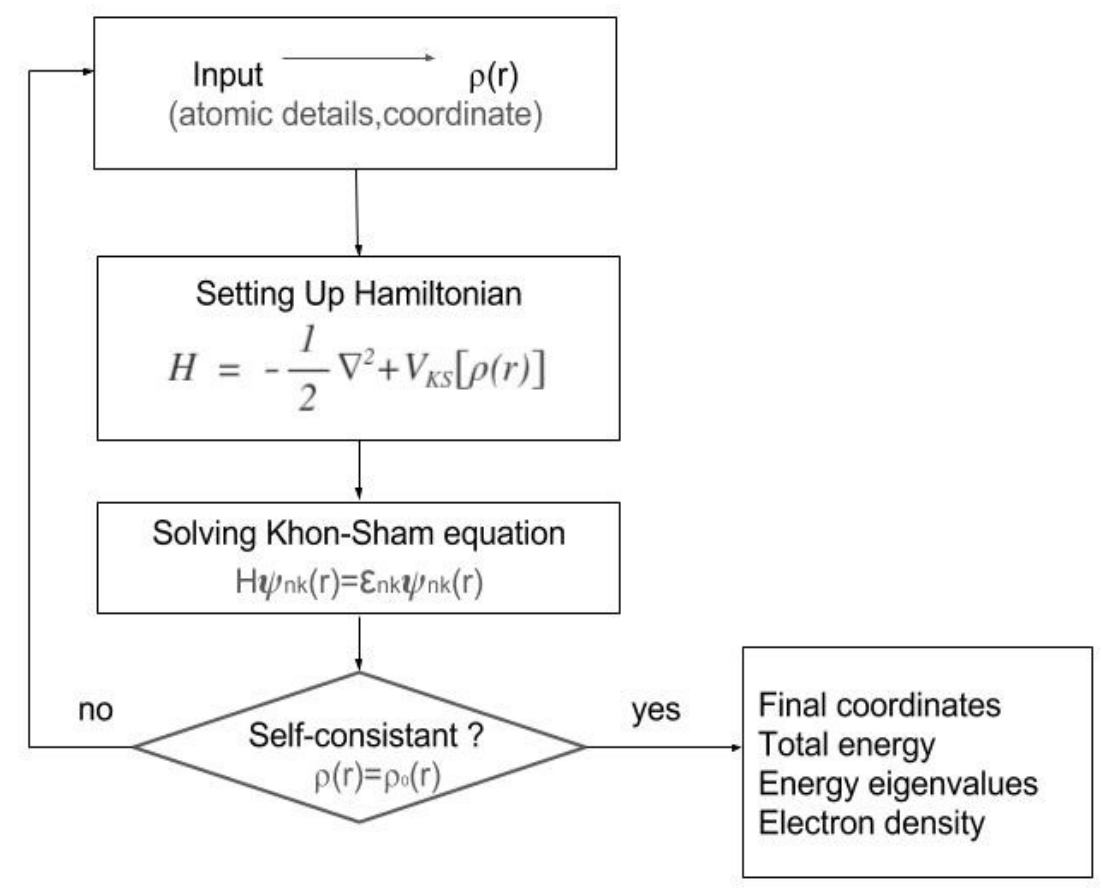

Figure 2.1: Self-consistent algorithm of DFT

extremely high precision. So that $E_{X C}$ depends only on the local electron density around each volume element $d r$ in the system. Exchange Correlation Functional can be represented as,

$$
E_{X C}[\rho]=\int \rho(r) \epsilon_{X C}[\rho(r)] d r
$$

where $\epsilon_{X C}[\rho(r)]$ is the energy density in homogeneous electron gas. It provides us with much successful results than expected, especially for solid, to describe the structural and vibrational properties. LDA yields results that compare well to HF results even for molecules and atoms. LDA is computationally much simpler than HF with the true exchange potential. It gives the correct crystal structure which is usually found to have the lowest energy, bond lengths, bulk moduli, phonon frequencies which are accurate within a few percent.

\subsubsection{Generalised Gradient Approximation (GGA)}

As the LDA approximate the energy of the true density by the energy of a local constant density, it fails in the situations where the density undergoes rapid change such as in molecules. An improvement to this situation can be made by considering the gradient of the electron density, so called 
Generalised Gradient Approximation, symbolically it can be written as,

$$
E_{X C}^{G G A}=\int f\left[\rho(r), \nabla_{r} \rho(r)\right] d r
$$

This can lead to large improvement over LDA result. Some of these are semi-empirical, in that experimental data e.g atomization energy is useful in their derivation. A commonly used functional are PBE, PW91 functional, due to Predew-Burke-Ernzerhof parameterization, Perdew and Yan respectively.

\subsubsection{Tran-Blaha modified Becke-Johnson potential (TB-mBJ)}

Even though traditional functions like LDA and GGA produce reasonably good results, they underestimate the band gaps of many semiconductors and insulators. Tran-Blaha modified Becke-Johnson potential (TB-mBJ) can be one of the solutions to this problem since this exchange correlation function can capture the exact band gap. This is used in the present thesis in order to accurately determine the band gaps of the investigated compounds. Tran-Blaha modified Becke-Johnson potential is given by,

$$
v_{x, \sigma}^{T B-m B J}(r)=c v_{x, \sigma}^{B R}(r)+(3 c-2) \frac{1}{\pi} \sqrt{\frac{5}{6}} \sqrt{\frac{t_{\sigma}(r)}{\rho_{\sigma}(r)}}
$$

Where $\rho_{\sigma}=\sum_{i=1}^{N_{\sigma}}\left|\psi_{i, \sigma}\right|^{2}, t_{\sigma}=\frac{1}{2} \sum_{i=1}^{N_{\sigma}} \nabla^{*} \psi_{i, \sigma} . \nabla^{*} \psi_{i, \sigma}, v_{x, \sigma}^{B R}(r)=-\frac{1}{b_{\sigma}(r)}\left(1-e^{-x_{\sigma}(r)}-\frac{1}{2} x_{\sigma}(r)\left(e^{x_{\sigma}(r)}\right)\right)$, are the electron density, kinetic energy density and the Becke-Roussel (BR) exchange potential respectively here $x_{\sigma}$ is obtained from a non-linear equation involving $\rho_{\sigma}, \nabla \rho_{\sigma}, \nabla^{2} \rho_{\sigma}$ and $t_{\sigma}$. The $b_{\sigma}$ is calculated with $b_{\sigma}=\left[x_{\sigma}^{3} e^{x_{\sigma}} /\left(8 \pi * \rho_{\sigma}\right)\right]^{(1 / 3)}$. Value of $c$ is $c=\alpha+\beta\left(\frac{1}{V_{\text {cell }}} \int_{\text {cell }} \frac{\nabla \rho\left(r^{\prime}\right)}{\rho\left(r^{\prime}\right) d^{3} r^{\prime}}\right)$. Where $V_{\text {cell }}$ is the unit cell volume and $\alpha$ and $\beta$ are two free parameters whose values are $\alpha=-0.012$ and $\beta=1.023 b h_{o r}{ }^{(1 / 2)}$. Using the TB-mBJ functional we have calculated electronic structure properties and further evaluated the thermoelectric properties.

\subsection{Introduction to Thermoelectric Materials}

Thermoelectric materials are considered to be one of the ideal solutions for sustainable energy. Since they can convert heat directly into electricity and vice-versa they are seen as green energy source because of its ability to convert waste heat into electricity. Development of new materials and fabrication technologies enable us to explore more about these particular class of materials to improve their efficiency and this objective became a key issue in the research field. In solids electrons not 
only conduct electricity but also conduct heat. These two phenomenon are coupled, since electrical conductivity transport energy and thermal conductivity transport heat by means of charge from hot junction to cold junction. Thus this coupling between electrical and thermal transport give rise to thermoelectric phenomenon $[55,56]$. Thermoelectric materials show thermoelectric effect i.e either a temperature difference can create an electric potential or a potential difference can create a temperature difference and their major application lies in (micro) cooling or electricity generation from heat sources. There are three types of thermoelectric effect. Seebeck Effect, Peltier Effect and Thomson Effect.

\section{Seebeck effect}

If two wires of dissimilar metals are joined end to end and two junctions are maintained at different temperature i.e connected electrically in series and thermally in parallel, then a current flows through the circuit. This is known as Seebeck Effect. Such a current is known as thermoelectric current and emf producing it is called thermo-emf and was first reported by Thomas Seebeck in 1821. The circuit formed by the two wires of dissimilar metals is called thermocouple. The direction and magnitude of the Seebeck voltage (V), depends on the temperature difference between the two junctions of the thermocouple and on the materials making up the thermocouple i.e on the Seebeck coefficient[57]

\section{Peltier Effect}

If a current is made to flow through the circuit of two dissimilar metals by using an external source then one junction gets heated whereas other gets cooled, depending on the direction of current flow, this phenomenon is known as Peltier Effect[55], the heat evolved or absorbed as Peltier heat. The rate of heating or cooling at a junction is found to be proportional to the strength of the current and changes its sign on reversing the direction of current. Thus Peltier effect is reversible. The effect is just the reverse of the Seebeck Effect. Thus materials exhibiting a large Seebeck effect also show a large Peltier effect. The effect can be quantitatively described by the Peltier coefficient $\pi$. The Peltier coefficient $(\pi)$ is determined by the ratio of the rate of heating $(\mathrm{Q})$ to the current $(\mathrm{I})$.

\section{Thomson Effect}

In order to connect the thermoelectric effects as observed by Seebeck and Peltier, William Thomson described the third thermoelectric effect, Thomson effect which describes the resulting electric current that develops in a single conductor when a small temperature gradient is applied [29-31]. This relationship is described by the equation $Q=\beta I \triangle T$, where $Q$ is the rate of heating, $I$ is electric 
current, $\triangle T$ is change in temperature, and $\beta$ is the Thomson coefficient. This relationship holds if the temperature difference, $\triangle T$, is small. 


\section{Chapter 3}

\section{Methodology}

All the total energy calculations based on first principle density functional theory (DFT) were performed using pseudopotential method as implemented in the Plane wave self-consistent field (Pwscf) program [18] and full-potential linear augmented plane wave (FP-LAPW) method as implemented in the WIEN2k [19]. The Pwscf method is used to perform the structural optimization, whereas FP-LAPW method is used to study the electronic, mechanical and transport properties. The total energies are obtained by solving the Kohn-Sham equation self consistently within the Generalized Gradient Approximation (GGA) of Perdew-Burke-Ernzerhof (PBE) potential [20]. A plane wave kinetic energy cut-off of $50 \mathrm{Ry}$ is used and the first Brillouin zone is sampled according to the Monkhorst-Pack scheme [21] by means of a $8 \times 8 \times 8 \mathrm{k}$-mesh in order to ensure that the calculations are well converged. Since the traditional functionals such as local density approximation and generalised gradient approximation methods were underestimating the band gap, we adopted Tran-Blaha modified Becke-Johnson potential (TB-mBJ)[22, 23] to attain a proper band gap for the investigated compounds. TB-mBJ is found to be quite successful in reproducing the experimental band gaps as compared to standard GGA $[22,24,25,26,27]$. Considering the presence of heavy elements, we have included spin orbit coupling in our calculations. All the calculations were performed with the optimized lattice parameters with an energy convergence criterion of $10^{-6}$ Ry per formula unit.

The carrier concentration ( $\mathrm{p}$ for holes and $\mathrm{n}$ for electrons) dependent transport properties like thermopower $(S)$, electrical conductivity scaled by relaxation time $(\sigma / \tau)$ and power factor $\left(S^{2} \sigma / \tau\right)$ were calculated using the BoltzTraP[28] code. This fraction of code is an implementation of semiclassical Boltzmann transport equation using constant relaxation-time approximation (CSTA) and rigid band approximation (RBA). The detailed explanation about the CSTA is given in Ref. [29, 
31, 30] and the references cited therein. It is evident that CSTA has been quite successful in the past in predicting the thermoelectric properties of many materials [32, 33, 34, 30, 35]. According to the RBA approximation, doping a system does not alter its band structure but varies only the chemical potential, and it is a good approximation for doped semiconductors to calculate the transport properties theoretically when doping level is not very high [36, 37, 38, 39, 40]. However certain types of dopant can drastically modify the nature of electronic structure near the gap giving rise to resonant states in which case the RBA can fail [41]. The crystal structures are generated using the VESTA [42] software and the charge density plots are generated with the help of Xcrysden molecular structure visualization program [43]. 


\section{Chapter 4}

\section{Results and Discussions}

\subsection{Structural and Mechanical properties}

The investigated compounds $\mathrm{CaLi} P n(P n=\mathrm{As}, \mathrm{Sb}, \mathrm{Bi})$ crystallize in orthorhombic structure with space group Pnma(62), and the crystal structure is given in Fig. 4.1. In Table 4.1 we have presented the optimized ground state properties along with available experimental reports. From Table 4.1, it is quite evident that the calculated optimized parameters are in good agreement with the experimental reports.

Elastic constants are the fundamental material parameters that describe the resistance of the material against applied mechanical deformation. This will also indicate the mechanical stability of the investigated compounds. All the investigated compounds crystallise in an orthorhombic structure and it has nine independent elastic constants, namely, $C_{11}, C_{22}, C_{33}, C_{44}, C_{55}, C_{66}, C_{12}$, $C_{13}$, and $C_{23}$. The calculated single-crystal elastic constants at the theoretical equilibrium volume

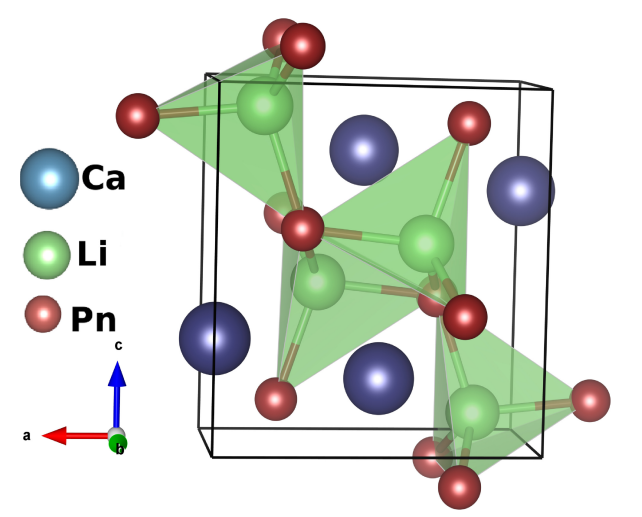

Figure 4.1: Crystal structure of $\mathrm{CaLi} P n(P n=\mathrm{As}, \mathrm{Sb}, \mathrm{Bi})$ 
Table 4.1: Calculated structural parameters of CaLiPn ( $P n=\mathrm{AS}, \mathrm{Sb}, \mathrm{Bi})$ along with the experimental [16] parameters

\begin{tabular}{|ccccccc|}
\hline Compounds & CaLiAs & & CaLiSb & & CaLiBi & \\
\hline & Present & Exp & Present & Exp & Present & Exp \\
\hline $\mathrm{a}($ in $\AA)$ & 7.289 & 7.233 & 7.693 & 7.642 & 7.832 & 7.729 \\
$\mathrm{~b}($ in $\AA$ ) & 4.334 & 4.313 & 4.655 & 4.632 & 4.750 & 4.713 \\
$\mathrm{c}($ in $\AA)$ & 7.945 & 7.895 & 8.310 & 8.292 & 8.497 & 8.422 \\
\hline
\end{tabular}

are tabulated in Table 4.2 .

Table 4.2: Elastic constant $\left(C_{i j}\right)$, Young modulus, Bulk modilus (in GPa), sound velocities $\left(v_{l}, v_{t}\right.$, $\left.v_{m}, \mathrm{~km} / \mathrm{sec}\right)$ and Debye Temperature $\left(\Theta_{D}, \mathrm{~K}\right)$ of $\mathrm{CaLi} P n$

\begin{tabular}{|cccc|}
\hline Elastic Constant & CaLiAs & CaLiSb & CaLiBi \\
\hline $\mathrm{C}_{11}$ & 100.50 & 81.69 & 64.93 \\
$\mathrm{C}_{22}$ & 104.95 & 89.25 & 72.64 \\
$\mathrm{C}_{33}$ & 79.23 & 73.59 & 58.75 \\
$\mathrm{C}_{44}$ & 45.78 & 37.46 & 31.21 \\
$\mathrm{C}_{55}$ & 35.37 & 31.39 & 24.02 \\
$\mathrm{C}_{66}$ & 38.73 & 33.11 & 24.71 \\
$\mathrm{C}_{12}$ & 16.25 & 17.15 & 12.44 \\
$\mathrm{C}_{13}$ & 17.39 & 17.09 & 13.11 \\
$\mathrm{C}_{23}$ & 25.11 & 21.55 & 18.35 \\
Young modulus & 89.88 & 77.09 & 61.0686 \\
Bulk modulus & 44.43 & 39.44 & 31.45 \\
v $_{\text {Longitudinal }}$ & 5.57 & 4.81 & 3.59 \\
v $_{\text {Transverse }}$ & 3.48 & 2.97 & 3.16 \\
v $_{\text {Mean }}$ & 3.83 & 3.27 & 2.43 \\
Debye Temperature & 411.2 & 331.1 & 242.6 \\
\hline
\end{tabular}

All the calculated single-crystal elastic constants satisfied the Born's mechanical stability criteria for orthorhombic structure [44] thereby implying that the investigated compounds are mechanically stable under ambient conditions. By using the calculated single-crystal elastic constants, we have further computed the polycrystalline aggregate properties such as bulk moduli $\left(B_{X}, X=V, R\right.$, or $\left.H\right)$, shear moduli $\left(G_{X}, X=V, R, o r H\right)$ using the Voigt, Reuss, and Hill approaches. Apart from these, we have also calculated the Debye temperature $\left(\Theta_{D}\right)$ using sound velocities. $\Theta_{D}$ is a fundamental quantity that correlates several physical properties such as specific heat, thermal conductivity, and melting point of the crystal with elastic constants. At low temperatures, $\Theta_{D}$ can be estimated from the average sound velocity $\left(v_{m}\right)$, which is the average of longitudinal $\left(v_{l}\right)$ and $\operatorname{transverse}\left(v_{t}\right)$ sound velocities. The calculated values of $v_{l}, v_{t}, v_{m}$ and $\Theta_{D}$ are shown in Table 4.2. This is the first qualitative prediction of the mechanical properties of zintl phase CaLiPn. As the investigated compounds are newly synthesised [16], there are no much information available in the literature to 
compare the mechanical properties of the present studied compounds. But we tried to compare the elastic constant coefficients of the present investigated compounds with the prototype family of SrLiAs material [17]. It is found that in SrLiAs $C_{11}>C_{22}<C_{33}$, and the same order is also observed in all the investigated compounds of $\mathrm{CaLiP} n$. Further we also find that the order of Debye temperature of SrLiAs and the investigated compounds of CaLiPn are almost similar. This indicates that the investigated compounds might also possess low thermal conductivity in comparison with SrLiAs.

\subsection{Electronic properties}

Optimised lattice parameters from Pwscf are used further to calculate electronic and transport properties. The electronic structure of $\mathrm{CaLi} P n(P n=\mathrm{As}, \mathrm{Sb}, \mathrm{Bi})$ compounds were calculated using TB-mBJ functional. The band structure of all the investigated compounds along the high symmetry directions of the Brillouin zone are presented in Fig. 4.2.

It can be observed that all the compounds are found to be direct band gap semiconductors since the valence band maximum and conduction band minimum are located at the centre of the Brillouin zone. The band gaps for CaLiAs, CaLiSb and CaLiBi are $1.57 \mathrm{eV}, 0.89 \mathrm{eV}$ and $0.6 \mathrm{eV}$ respectively. The calculated band gaps are found to decrease from CaLiAs to CaLiBi. The calculattions using TBmBJ method is found to be fairly accurate in these kind of zintl phase materials such as SrLiAs[17] which has similar structure and similar space group as CaLiPn. The band dispersion along three crystallographic direction $\Gamma-\mathrm{X}, \Gamma-\mathrm{Y}$ and $\Gamma-\mathrm{Z}$ of $\mathrm{CaLi} P n$ are found to be having similar pattern in valence band for all three compounds. In the valence band and conduction band the dispersion along $\Gamma-\mathrm{Y}$ is found to be more compared to other two axes for all the compounds. Because of this higher dispersion, $\Gamma-Y$ direction will have less effective mass compared to other directions. The principal aim of the present work is to calculate the thermoelectric properties of $\mathrm{CaLiPn}$ and to study its variation with carrier concentration. It is necessary to estimate the effective masses of the carriers in various electron and hole pockets at the band edges. We have calculated the mean effective mass of the carriers at the conduction and valence band edges by fitting the energy of the respective bands to a quadratic polynomial in the reciprocal lattice vector $\vec{k}$. The calculated effective masses for CaLiPn in crystallographic directions of the Brillouin zone are tabulated in Table 4.3.

It can be observed from the table that for all three compounds, the calculated effective masses in the $\Gamma$-Y direction for the valence bands are $m_{y}^{*}=4.5 m_{e}, m_{y}^{*}=3.59 m_{e}$, and $m_{y}^{*}=2.58 m_{e}$ for CaLiAs, CaLiSb and CaLiBi respectively. These values are low when compared with other directions which is expected because of the high dispersion bands along the $\Gamma-Y$ in all the compounds. The 


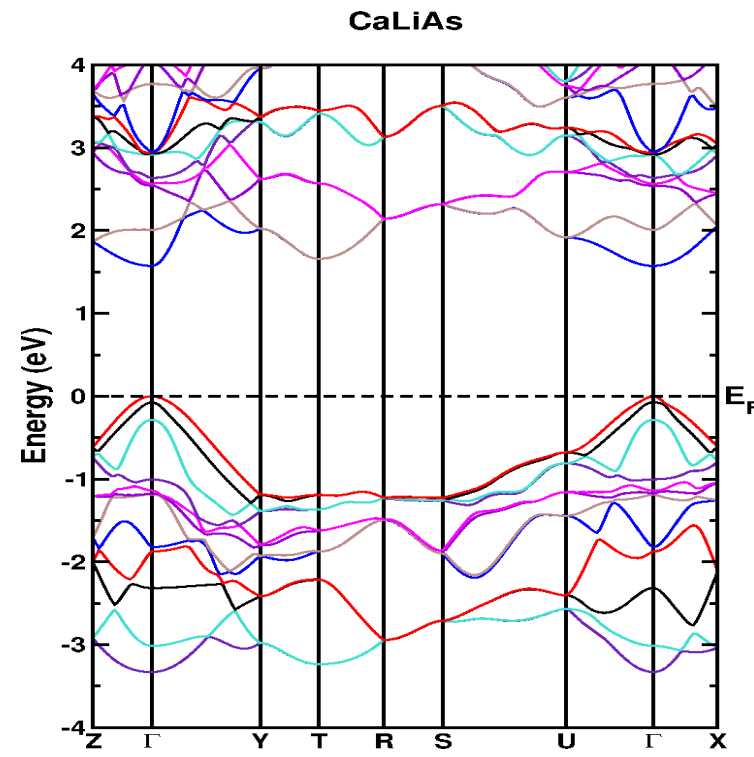

(a)

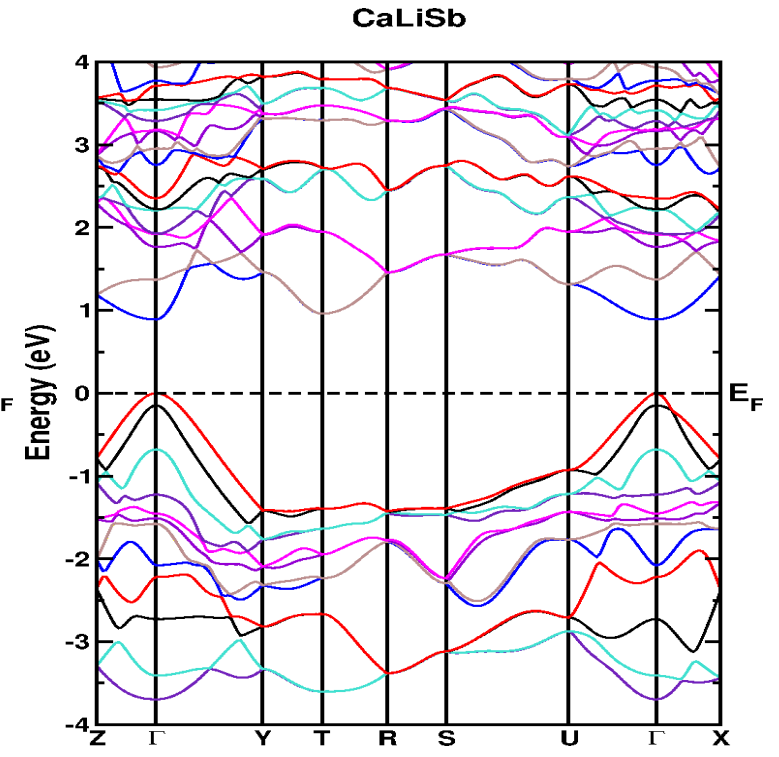

(b)

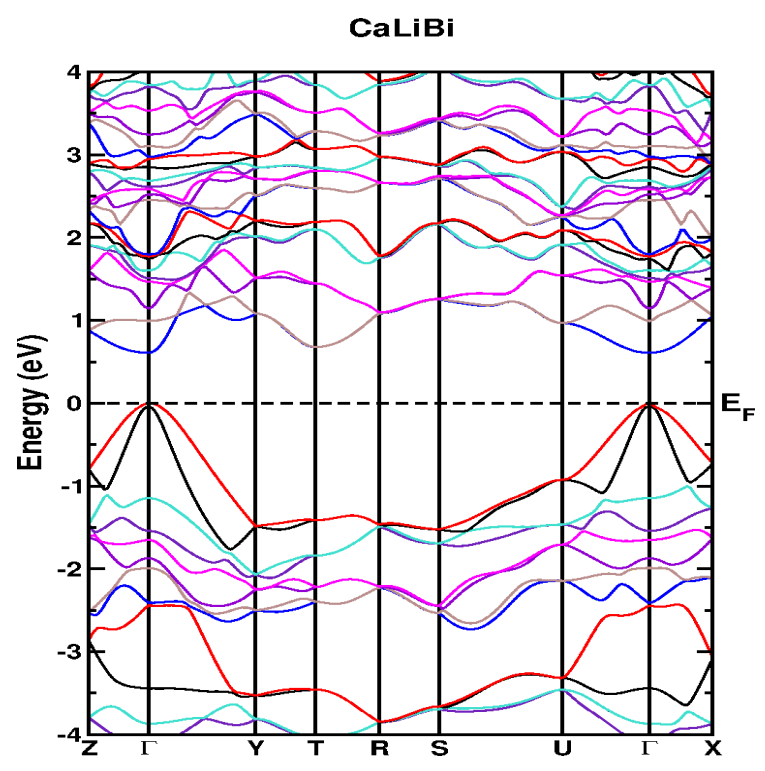

(c)

Figure 4.2: Calculated band structure of (a) CaLiAs (b) CaLiSb and (c) CaLiBi 
Table 4.3: Calculated effective mass of CaLiPn in crystallographic directions of the Brillouin zone are given in the units of electron rest mass

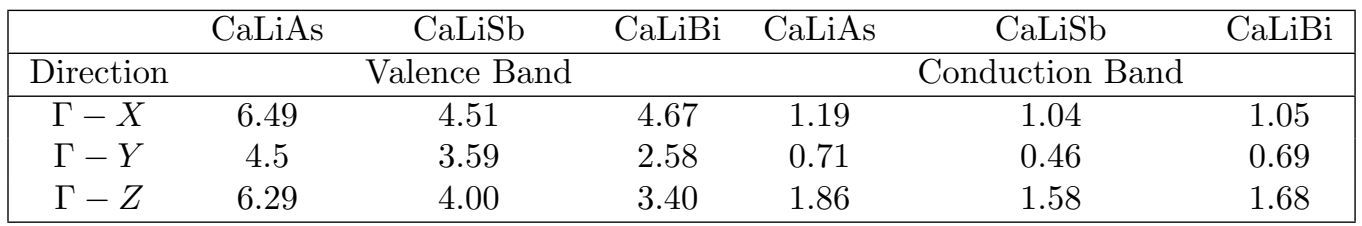

values of effective masses of conduction bands also follow the same trend as that of the valence band. The flat bands are observed along $R-S$ high symmetric direction.

Further analysis of the electronic properties requires the total and partial density of states of these compounds, and the same is represented in Fig 4.3. In the valence band anion Pn states are dominating and in the conduction band Ca-states are dominating for all the three compounds. From CaLiAs to CaLiBi, the contribution of Li-s states are found to be increased near the Fermi level. The heavy band found just below the valence band maximum arises from the $P n-p$ states, and the light band lying just below is contributed mainly by Ca- $p$ and Li-s states. Compared to valence band, more hybridised Ca states are observed in the conduction band. Further we have also calculated the charge density of CaLiAs to analyse the bonding nature of the investigated compounds and the same is presented in Fig. 4.4. From this figure it is evident that there exists covalent nature between Li-As. In general the weak electro-negativity of 'Li' atom will form the ionic bond in most of the cases $[10,11,12]$, but in the present case it is making a covalent bond as similar to SrLiAs [17]. The covalent bond bewtween Li-As reduces the mass of the carriers at the band edge resulting in light carriers. The heavy bands usually contribute to high thermopower since it is proportional to effective mass $\left(m^{*}\right)[13]$, while the lighter bands donate to high mobility (mobility, $\mu=\tau e / m^{*}$, where $e$ is the charge). A favourable combination of the two may lead to an excellent TE performance in materials[5]. For CaLiAs, the variation of density of states near Fermi level is almost similar for valence band and conduction band, whereas in the case of other two compounds the change in density of states near Fermi level is more in conduction band than in valence band. This behaviour indicate that for CaLiAs, the TE properties for holes and electrons might be similar, whereas for the other two the electron doping will be more favourable.

\subsection{Thermoelectric properties}

In this section we have presented the thermopower (in $\mu V K^{-1}$ ) and electrical conductivity scaled by relaxation time ( in $\Omega^{-1} m^{-1} s^{-1}$ ), as a function of carrier concentration and temperature, using the 


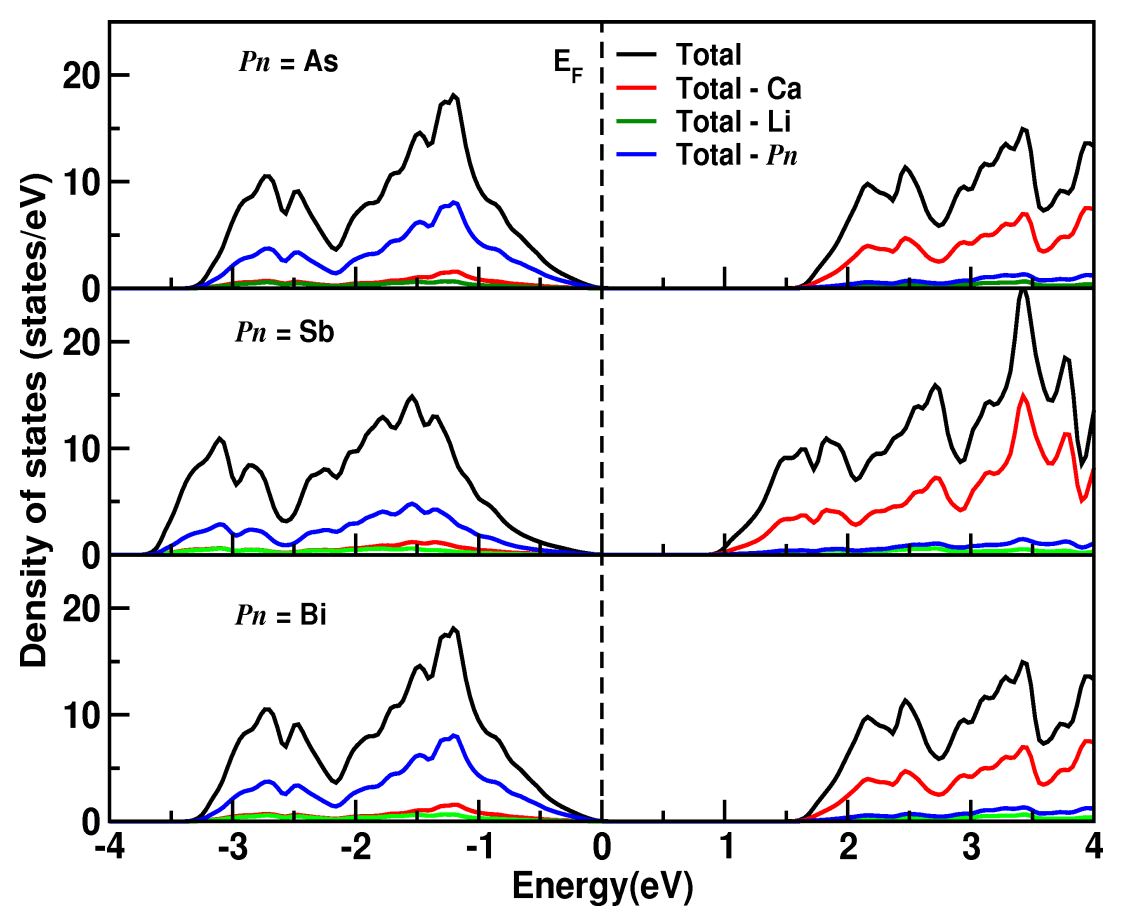

(a)

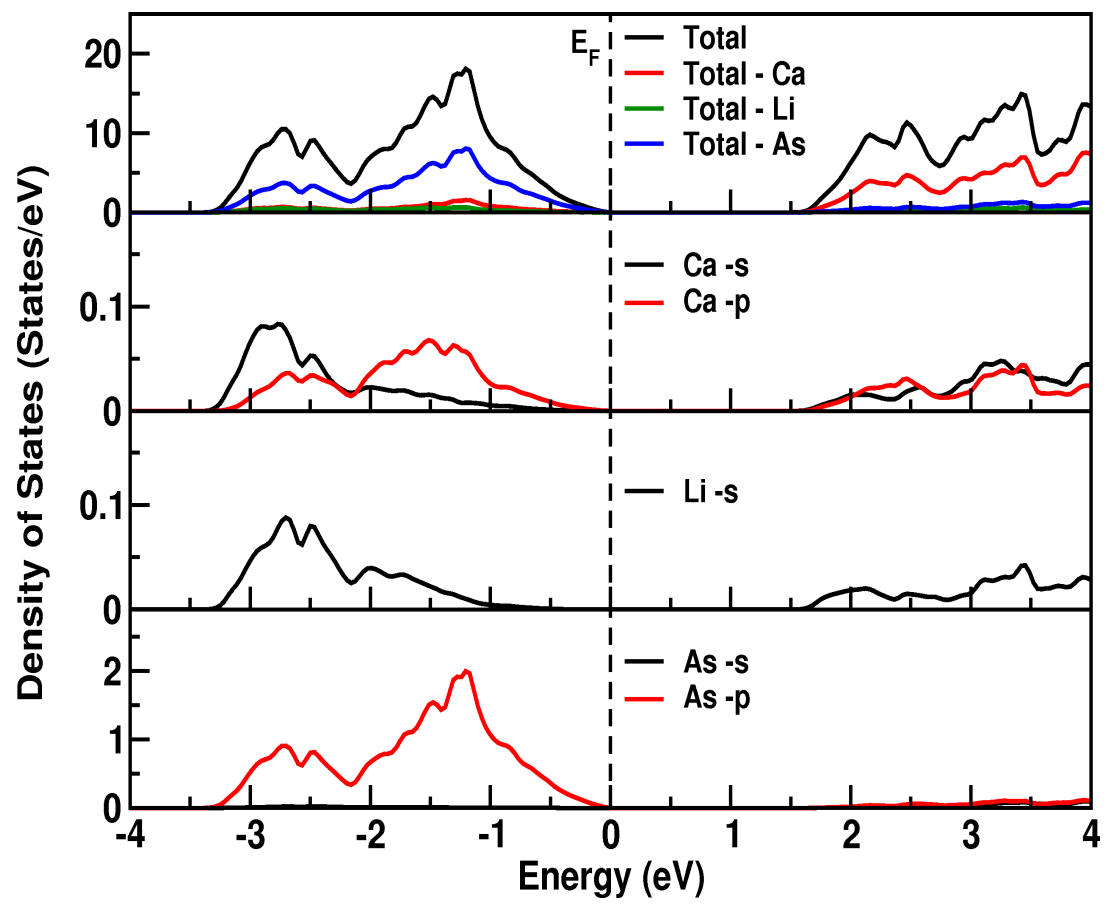

(b)

Figure 4.3: Calculated density of states of (a) CaLiPn ( $P n=\mathrm{As}$, Sb and Bi) (b) partial density of states of CaLiAs 


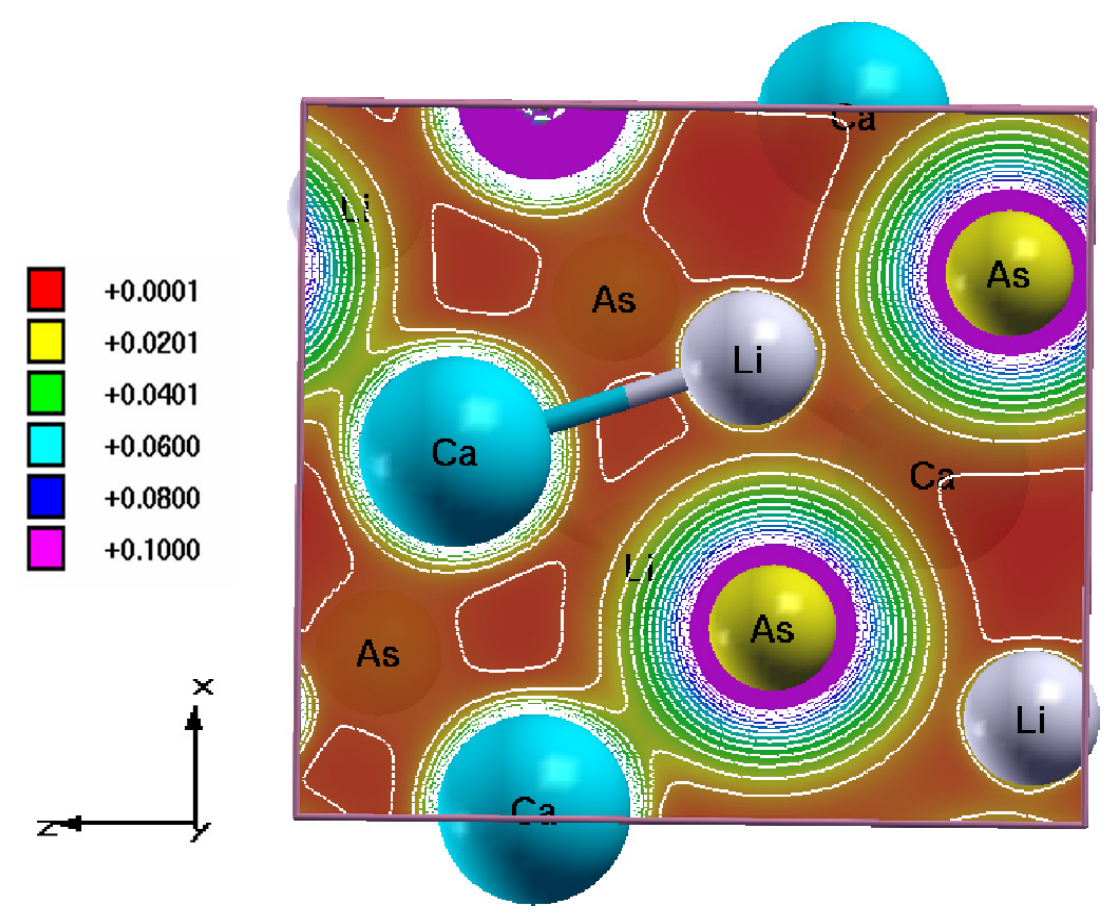

Figure 4.4: Calculated charge density of CaLiAs

Boltzmann transport equation approach as implemented in BoltzTraP [28] code. As the investigated compounds crystallise in orthorhombic structure, we have calculated TE properties along three crystallographic directions. The variation of thermoelectric properties of the investigated compounds as a function of temperature at concentrations around $10^{19}$ and $10^{20} \mathrm{~cm}^{-3}$ for both electrons and holes, along different crystallographic directions are reported in Fig. 4.5-4.7.

The thermopower of all the investigated compounds are found to be higher for the electrons compared to holes throughout the studied temperature range. The difference in the thermopower between electrons and holes is of the order of $150-200 \mu \mathrm{V} / \mathrm{K}$ for all the investigated compounds through out the studied temperature range. The higher values in the case of electrons as carriers might be due to the increased number of minima in the conduction band region compared to the valence band [49]. In the case of thermopower, almost isotropic behavior is observed along different crystallographic directions and this might be due to the similar band structure nature along different crystallographic direction. Thermopower is found to decrease as we move from As to Sb, which is due to the decreasing band gaps as we move from As to $\mathrm{Sb}$ (see in section 3-B). Among the three investigated compounds, n-type CaLiAs has more thermopower than the other two compounds. At high temperature above $800 \mathrm{~K}$, for CaLiBi we have observed bipolar conductivity (containing both free electrons and holes) which may lead to low, compensated thermopower and therefore low ZT, 

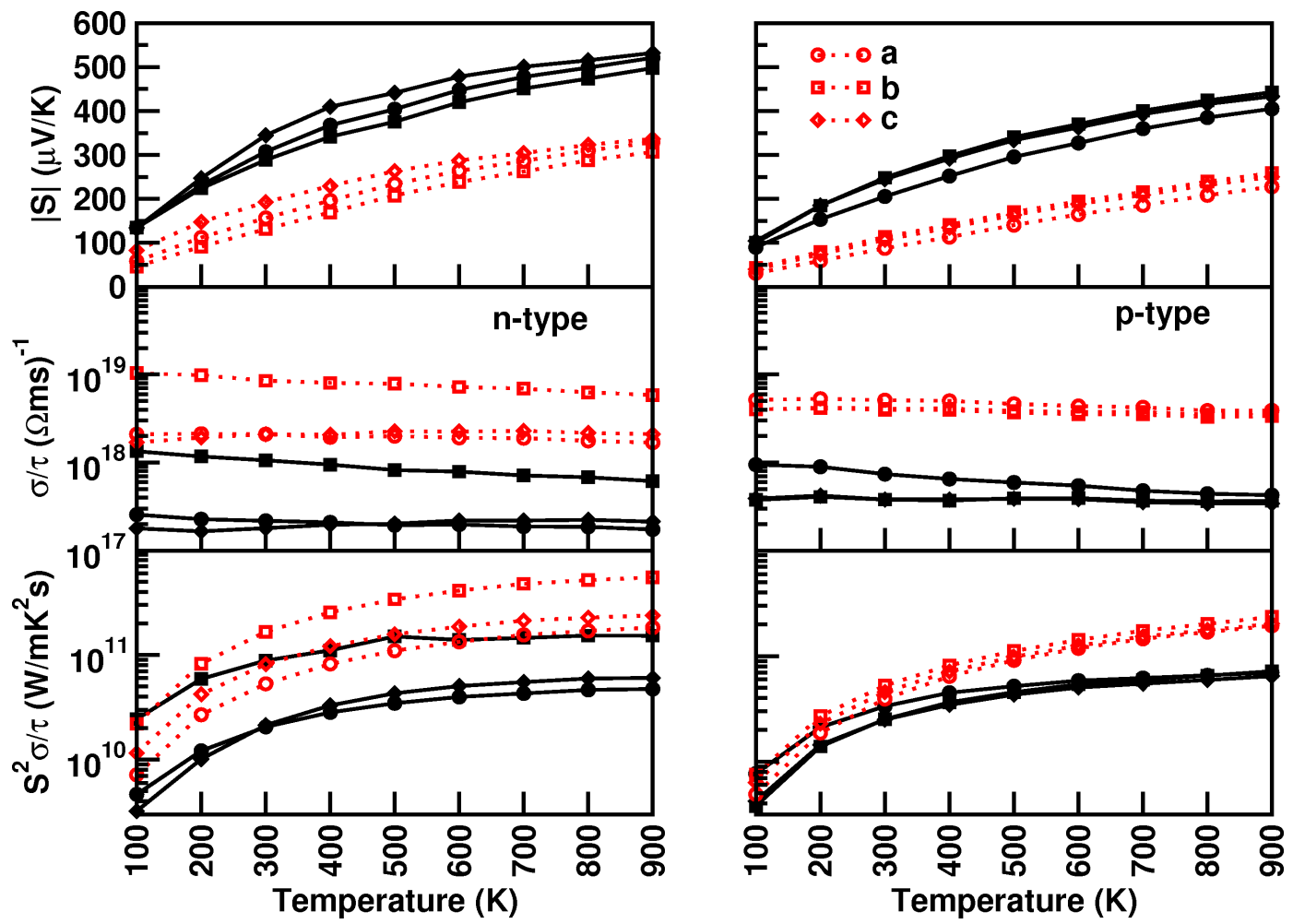

Figure 4.5: Calculated thermoelectric properties of CaLiAs for both electron and hole concentration at $10^{19}$ (solid lines with filled symbols) and $10^{20} \mathrm{~cm}^{-3}$ (dotted lines with open symbols) as function of temperature 

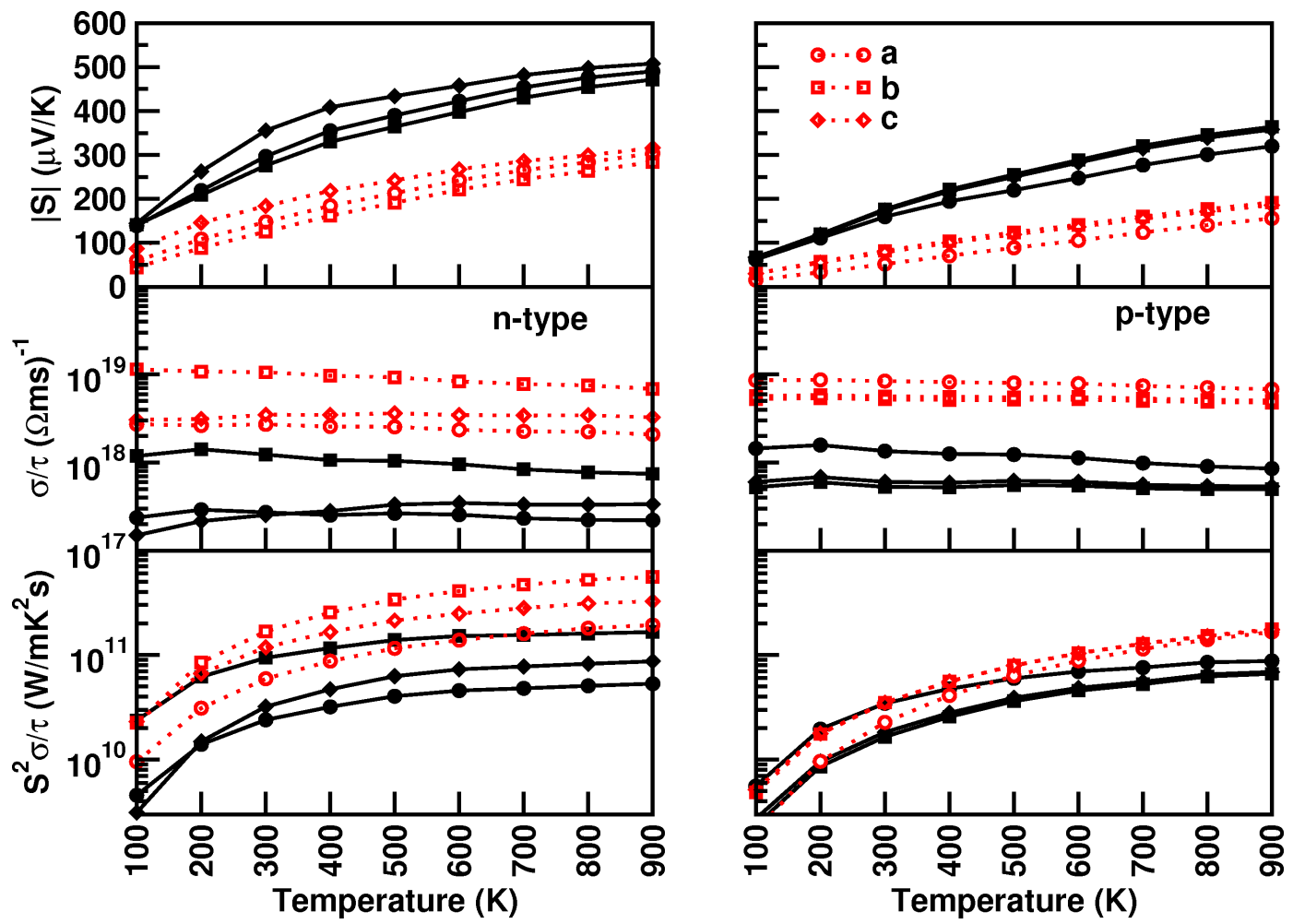

Figure 4.6: Calculated thermoelectric properties of CaLiSb for both electron and hole concentration at $10^{19}$ (solid lines with filled symbols) and $10^{20} \mathrm{~cm}^{-3}$ (dotted lines with open symbols) as function of temperature 

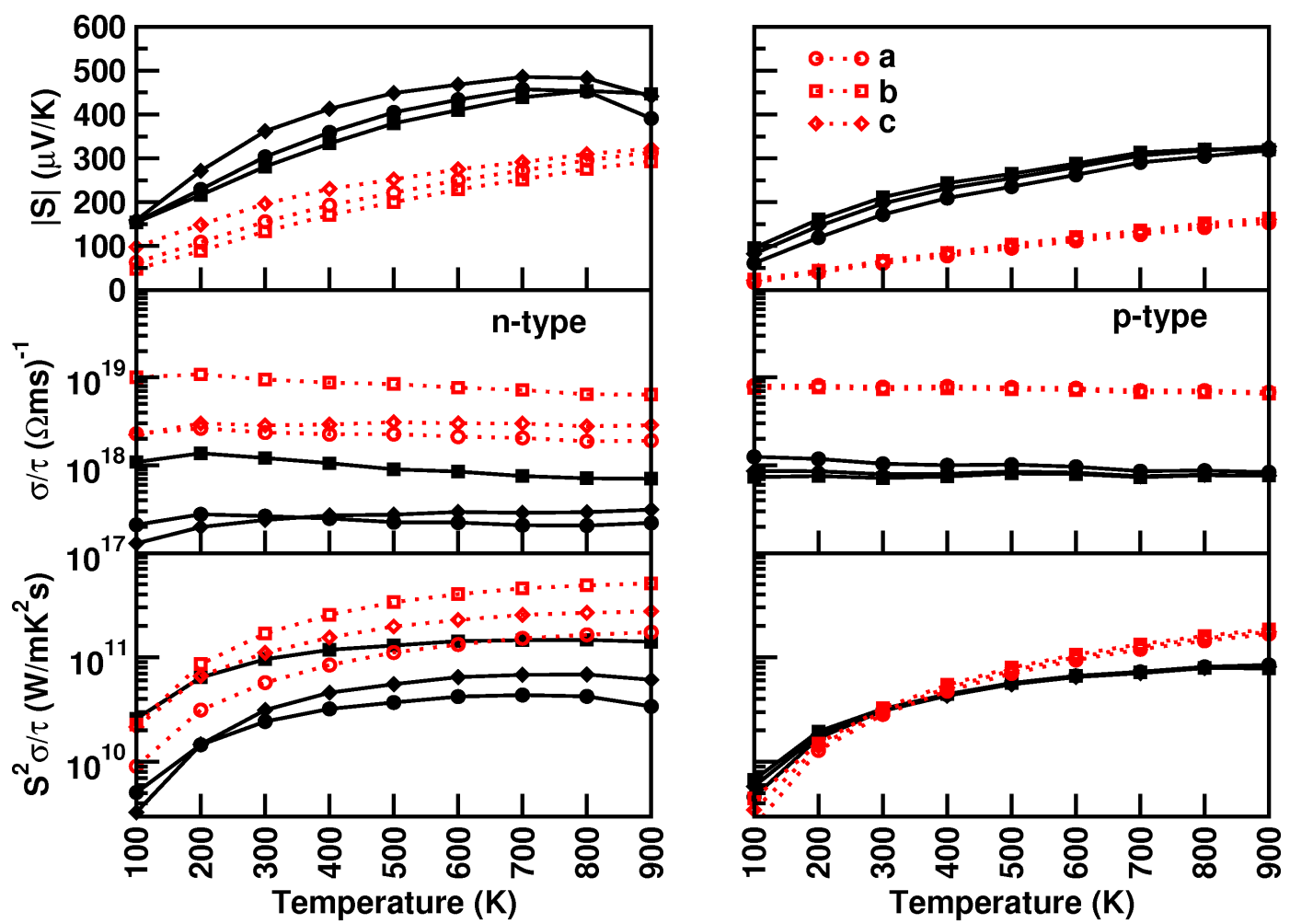

Figure 4.7: Calculated thermoelectric properties of CaLiBi for both electron and hole concentration at $10^{19}$ (solid lines with filled symbols) and $10^{20} \mathrm{~cm}^{-3}$ (dotted lines with open symbols) as function of temperature 
which restrict the performance of CaLiBi at high temperature.

Almost a similar type of variation is observed in the case of electrical conductivity scaled by relaxation time in all the compounds. All the investigated compounds have almost similar value of $\sigma / \tau$ at all the studied temperatures. Higher value of electrical conductivity is noticed in the case of electron concentration compared to holes, which is very similar to that of the thermopower of the investigated compounds. Further we find a considerable anisotropic nature in the case of electrons along the $b$-axis compared to the $a$ and $c$-axes. This might be due to the lower value of the effective mass along the $\Gamma-Y$ direction compared to other two directions in the case of conduction band. The strong dispersion along the $\Gamma-Y$ direction for all three compounds induced by the Li- $P n$ covalent bonding result in a low reduced mass and therefore increased conductivity along that direction. In the case of holes almost isotropic behavior in $\sigma / \tau$ is observed along different crystallographic directions. For further analysis, we have plotted the power-factor as a function of temperature. It is obvious that the power-factor for electron doping will be higher as compared to hole doping, because of the higher value of $S$ and $\sigma / \tau$ in the case of electrons and it is further noticed that $b$-axis power-factor will be higher due to the higher value of $\sigma / \tau$ along this direction. In the case of hole doping, the power-factor values are found to be almost isotropic along different crystallographic direction. Overall we can say that the electron doping is more favourable for TE properties for all the investigated compounds.

In order to analyse how better is the TE properties of the investigated compounds we have compared them with SrLiAs. For this we have calculated $S, \sigma / \tau$, and $S^{2} \sigma / \tau$ as a function of both electron and hole carrier concentration at $1000 \mathrm{~K}$ and the same is presented in Fig. 4.8.

As mentioned earlier electron doping is more favourable compared to holes in CaLiAs and a similar situation is also seen in the case of SrLiAs. From the Fig. 4.8, it is quite evident that the thermopower of CaLiAs is found to be little higher compared to SrLiAs. Almost similar value of $\sigma / \tau$ is observed in both the compounds. Further little higher value of the thermopower in the case of CaLiAs resulted in a higher value of the power-factor compared to SrLiAs. To conclude we find almost similar thermoelectric properties in both CaLiAs and SrLiAs, but more favourable case is observed with CaLiAs.

\subsection{Lattice dynamics}

Further we have studied the phonon dispersion of CaLiPn at ambient using DFPT [45]. The unit cell of CaLiPn contains 12 atoms and hence it has 36 phonon modes for each wave-vector, out of 


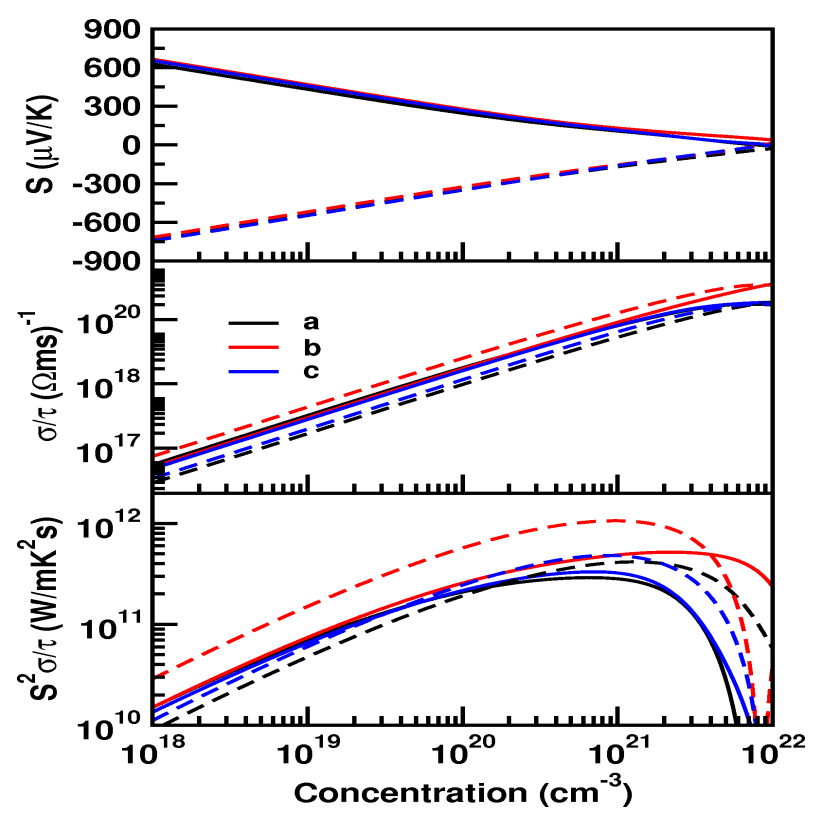

Figure 4.8: Calculated thermoelectric properties of CaLiAs as function of carrier concentration at $1000 \mathrm{~K}$

which three are acoustic and remaining modes are optical modes. The calculated phonon dispersion relation along with the phonon density of states of all three compounds are shown in Fig. 4.9. From the figure, it is clearly evident that the lower frequency modes originate mainly due to the vibrations of $P n$ atom, middle frequencies are due to $C a$ and finally the upper frequencies are from $L i$ atom. In the case of CaLiAs, low-frequency optical branches intersect the acoustic branches starting below $80 \mathrm{~cm}^{-1}$, below $60 \mathrm{~cm}^{-1}$ for CaLiSb and below $44 \mathrm{~cm}^{-1}$ for CaLiBi. From this observation it can be inferred that the phonon-phonon scattering is strong in all three compounds and comparatively more in CaLiSb and CaLiBi than CaLiAs. Because of these anharmonic lattice interactions, there is a coupling between different phonons which will limit the value of mean free path and thereby limiting thermal conductivity at temperatures relevant to thermoelectrics (mean free path of the particle $l=1 / T$, where $T$ is the temperature) [48, 39]. The feature of strong phonon-phonon scattering is also seen in other zintl phase materials [46, 47].

Further we tried to estimate the order of the thermal conductivity of the investigated compounds. For this purpose we have used analytical relation leading to the minimum thermal conductivity given by Clark $[50,51]$ and Cahill [52] model. According to these model the minimum thermal conductivity was given by,

$$
\begin{gathered}
\text { Clarke's model: } \kappa_{\text {min }}=0.87 k_{b} \bar{M}_{a}^{(-2 / 3)} E^{1 / 2} \rho^{1 / 6} \\
\text { Cahill's model: } \kappa_{\text {min }}=\frac{k_{B}}{2.48} n^{\frac{2}{3}}\left(v_{l}+2 v_{t}\right)
\end{gathered}
$$




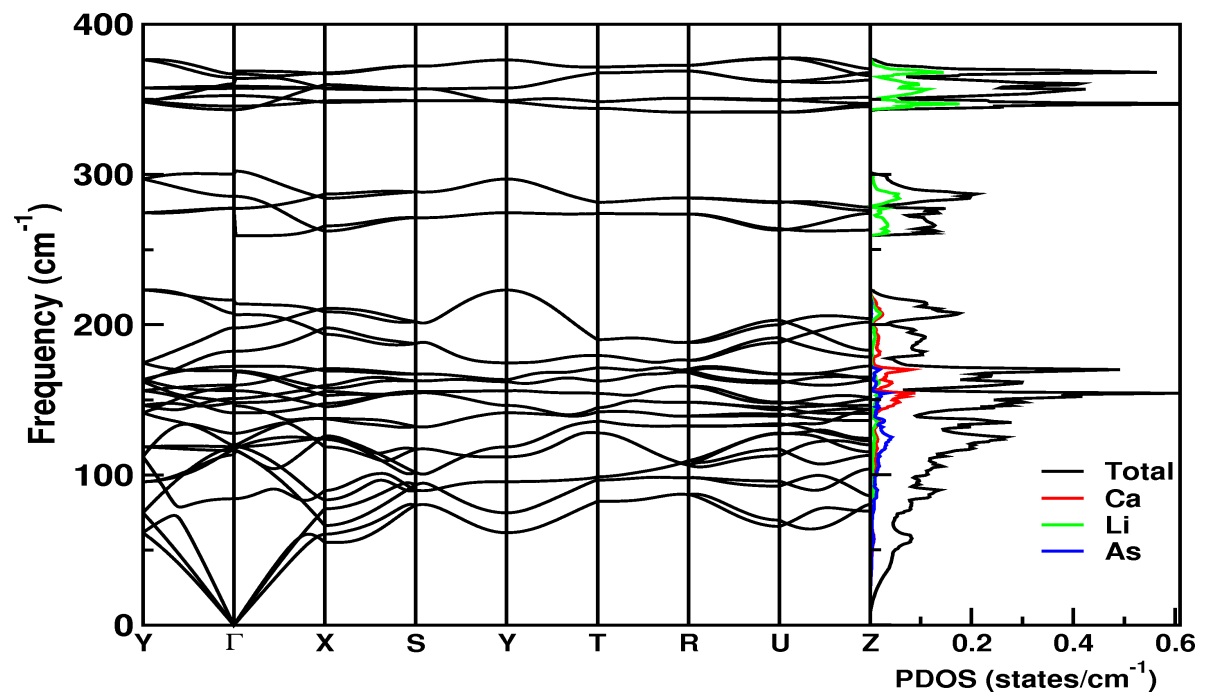

(a)

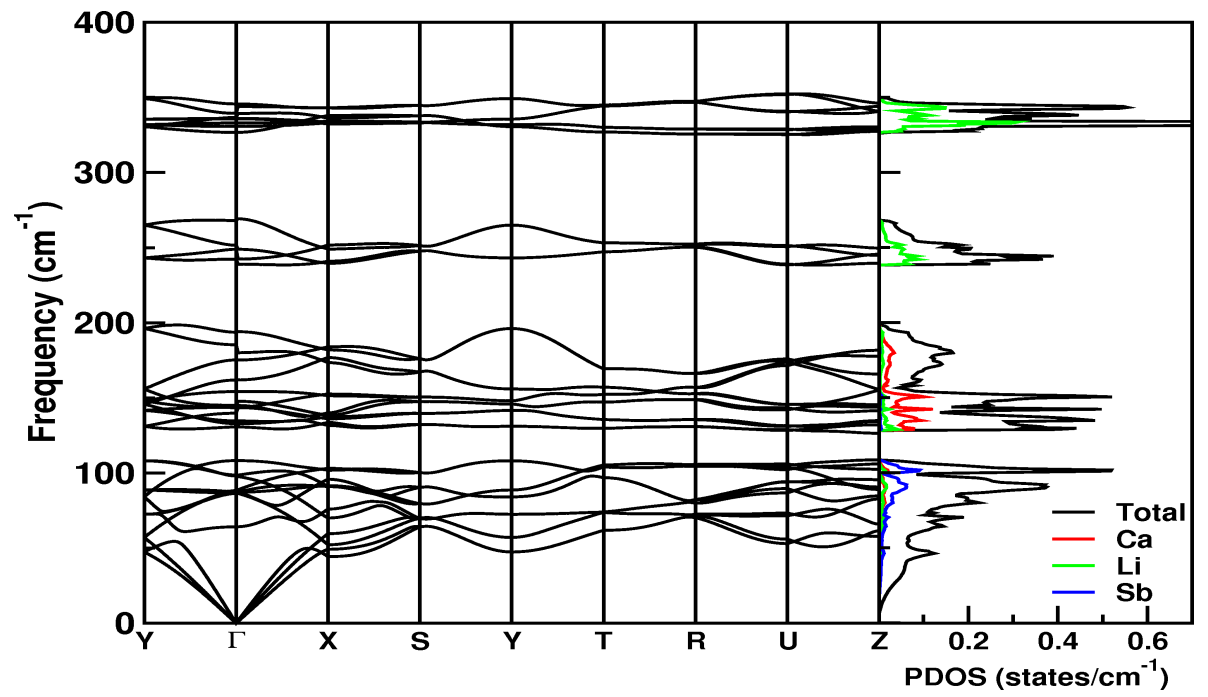

(b)

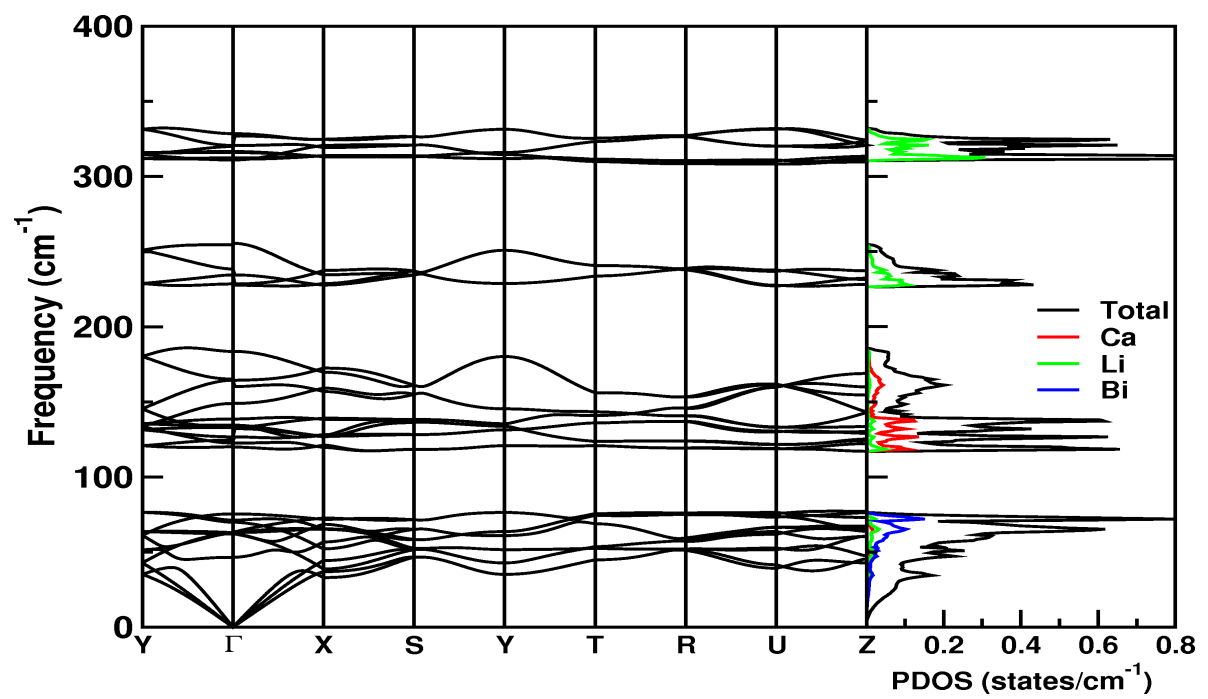

(c)

Figure 4.9: Calculated phonon dispersion of (a) CaLiAs (b) CaLiSb and (c) CaLiBi 
Here, $\bar{M}_{a}=\left[\frac{M}{\left(m \cdot N_{A}\right)}\right]$ is the average mass per atom, $E$ is the Youngs modulus, $\rho$ is the density, $M$ is the molar mass, $m$ is the total number of atoms per formula, $k_{B}$ is Boltzmanns constant, $N_{A}$ is Avogadros number, $n$ is the density of number of atoms per volume, and $v_{l}$ and $v_{t}$ are the average longitudinal and transverse sound velocities, respectively. These two methods are appropriate in predicting the minimum thermal conductivity for various materials $[53,54,17]$. In the present case we have also tried to predict the minimum thermal conductivity for all the investigated compounds and the same is presented in Table 4.4.

Table 4.4: Calculated minimum thermal conductivity of the investigated compounds of $\mathrm{CaLi} P n$ using the Clarke and Cahill models

\begin{tabular}{|lcc|}
\hline \multicolumn{3}{|c|}{$\kappa_{\min }$ in $\mathrm{W} / \mathrm{m} \mathrm{K}$} \\
\hline & Clarke Model & Cahill Model \\
\hline CaLiAs & 0.85 & 0.92 \\
CaLiSb & 0.65 & 0.71 \\
CaLiBi & 0.46 & 0.50 \\
\hline
\end{tabular}

To cross check with the calculated values, we also repeated the calculation of $\kappa_{m i n}$ for SrLiAs using both the methods. It has been shown that $\kappa_{m i n}$ is 0.65 and $0.70 \mathrm{~W} / \mathrm{m} \mathrm{K}$ for both Clarke and Cahill model, which is almost similar to the one presented in Ref.[17]. From the Table 4.4, it is quite evident that the $\kappa_{m i n}$ of the investigated compounds are found to be below unity. This confirms that investigated zintl phase Ca-based pnictides might show a promising TE performance. Except CaLiAs, other compounds are found to posses lesser $\kappa_{\min }$ compared to SrLiAs [17]. The calculated value of $\kappa_{\min }$ for the investigated compounds are also found to be in the same order of other Ca-based zintl phase compound $\mathrm{Ca}_{5} \mathrm{Al}_{2} \mathrm{Sb}_{6}$ [6]. This further confirms that the investigated Ca-based pnictide compounds are good TE candidates in comparison with SrLiAs. We look forward for the experimentalist to confirm the TE nature of Ca-based pnictides. 


\section{Chapter 5}

\section{Conclusion}

Zintl phase materials of $\mathrm{CaLi} P n(P n=\mathrm{As}, \mathrm{Sb}$ and $\mathrm{Bi})$ are investigated for the structural, mechanical, dynamical and transport properties using first-principles calculations. Two distinct density functional methods are used to investigate above properties. The plane wave pseudopotential approach was used to study the structural and dynamical properties. The full potential linear augment plane wave method has been used to study the electronic structure, mechanical and thermoelectric properties. The calculated structural properties of lattice parameters are in good agreement with available experimental data. The calculated electronic structure shows the investigated compounds to be direct band gap semiconductors. Further analysis of the electronic structure indicate the investigated compounds to possess mixed heavy and light bands, which is an indication to have good thermoelectric properties. We have also calculated the thermoelectric properties of all the investigated compounds for both carrier concentrations at various temperatures. We found a high thermopower for both the carriers, especially n-type doping is more favourable, which enabled us to predict that $\mathrm{CaLi} P n$ might have promising applications as a good thermoelectric material. Among the investigated compounds, we find that CaLiBi has bipolar conductivity at high temperatures which might be due to the lower band gap of this material compared to the rest of the compounds. Further the phonon dispersion curves of the investigated compounds reveals a flat phonon band, which will absorb more heat thereby resulting in low thermal conductivity. We have also observed the crossing of lower optical and acoustic branches which also further indicate that the investigated compounds may have an reasonable low thermal conductivities. The low value of the thermal conductivity is also confirmed through the empirical relation of Clarke and Cahill models, which has shown $\kappa_{\text {min }}$ to be below unity. With these findings, we predict that the investigated compounds are 
good thermoelectric materials which need to be examined further by the experimental study. 


\section{References}

[1] Ioffe A F 1957, Semiconductor Thermoelements and Thermoelectric Cooling, Translation by A. Gelbtuch and H. J. Goldsmid, Infosearch, London.

[2] Wood C 1988 Rep. Prog. Phys. 51459.

[3] Yang J H and Caillat T 2006 MRS.bull 31224

[4] Kauzlarich S M, Brown S R and Snyder G J 2007 Dalton Trans. 2099-2107

[5] Shakouri A 2011 Annu. Rev. Mater. Res 41399431.

[6] Toberer E S, Zevalkink A, Crisosto N, and Snyder G J 2010 Adv. Funct.Mater. 204375.

[7] Toberer E S, Cox C A, Brown S R, Ikeda T, May A F, Kauzlarich S M, and Snyder g J 2008 Adv. Funct. Mater. 182795.

[8] Zevalkink A, Pomrehn G, Takagiwa Y, Swallow J, and Snyder G J 2013 ChemSusChem. 102316.

[9] Kauzlarich S M, Zintl Compounds- In Encyclopedia of Organic Chemistry, 2nd ed.; King, R. B., Ed.; John Wiley and Sons: NewYork, 2005.

[10] Wang X L, Zeng Z, Ahn H, and Wang G X 2009 Appl. Phys. Lett. 95183103.

[11] Kaloni T P, Cheng Y C, Kahaly M U, and Schwingenschlogl U 2012 Chem. Phys. Lett. 53429.

[12] Kaloni T P, Balatsky A V, and Schwingenschlogl U 2013 EPL 10447013.

[13] Cutler M, Leavy J F and Fitzpatrick R L 1964 Electronic Transport in Semimetallic Cerium Sulfide, Phys. Rev. 1331143.

[14] Snyder G J and Toberer E S 2008 Nat. Mater. 7105.

[15] Toberer E S, May A F and Snyder G J 2010 Chem. Mater. 22624. 
[16] Schafer M C, Suen N and Bobev S 2014 Dalton Trans. 4316889.

[17] Guo L B, Wang Y X, Yan Y L, Yang G, Yang J M, and Feng Z Z 2014 J. Appl. Phys. 116 033705

[18] Baroni S, Gironcoli S D, dal Corso A et al $2008<$ http://www.pwscf.org $>$.

[19] Blaha P, Schwarz K, Madsen G K H, Kvasnicka D and Luitz J WIEN2k, An augmented plane wave + local orbitals program for calculating crystal properties (Karlheinz Schwarz, Techn. Universität Wien, Austria) 2001. < http://www.wien2k.at/>

[20] Perdew J P, Burke K, Ernzerhof M 1996 Phys. Rev. Lett. 77 3865-3868.

[21] Monkhorst H J, Pack J D 1979 Phys. Rev. B 13 5188-5192.

[22] Tran F, Blaha P, 2009 Phys. Rev. Lett. 102 226401-1-4.

[23] Becke A D, Johnson E R 2006 J. Chem. Phys. 124 221101-1-4.

[24] Koller D, Tran F, Blaha P 2011 Phys. Rev. B 83 195134-1-10.

[25] Koller D, Tran F, Blaha P 2012 Phys. Rev. B 85 155109-1-8.

[26] Dixit H, Saniz R, Cottenier S, Lamoen D, Partoens B 2012 J. Phys.: Condens. Matter 24 205503-1-9.

[27] Jiang H 2013 J. Chem. Phys. 138 134115-1-7.

[28] Madsen G K H and Singh D J 2005 Comput. Phys. Commun 67175.

[29] Singh D J 2010 Func. Mat. Letters. 3223.

[30] Ong K P, Singh D J and Wu P 2011 Phys. Rev. B 83115110.

[31] Parker D, Singh D J 2012 Phys. Rev. B 85 125209-1-7.

[32] David J, Singh, Mazin I I 1997 Phys. Rev. B 56 R1650-R1653.

[33] Parker D, Singh D J 2010 Phys. Rev. B 82 035204-1-5.

[34] Madsen G K H, Schwarz K, Blaha P and Singh D J 2003 Phys. Rev. B 68125212.

[35] Zhang L, Du M, Singh D J 2010 Phys. Rev. B 81 075117-1-8.

[36] Jodin L, Tobola J, Pécheur P, Scherrer H, Kaprzyk S 2004 Phys. Rev. B 70 184207-1-11. 
[37] Chaput L, Pécheur P, Tobola J, Scherrer H, 2005 Phys. Rev. B 72 085126-1-11.

[38] Bilc D I, Mahanti S D, Kanatzidis M G 2006 Phys. Rev. B 74 125202-1-12.

[39] Ziman J M 1960 Electrons and Phonons: The theory of transport phenomena in solids, Oxford University Press, Oxford.

[40] Nag B R 1980 Electron Transport in Compound Semiconductors (Springer-Verlag, Berlin).

[41] Lee M S and Mahanti S D Phys. Rev. B 85165149.

[42] Momma K and Izumi F 2011 J. Appl. Crystallogr. 4412721276.

[43] Kokalj A 2003 Comput. Mater. Sci. 28155.

[44] Born M, Huang K 1988 Dynamical Theory of Crystal Lattices, Oxford University Press: Oxford, U.K.

[45] Gonze X 1997 Phys. Rev. B 55 10337-10354.

[46] Gudelli V K, Kanchana V, Vaitheeswaran G, Singh D J, Svane A, Christensen N E, Mahanti S D 2015 Phy. Rev. B 92045206.

[47] Li L, Sui J, Pei Y, Barreteau C, Berardan D, Dragoe N, Cai W, He J and Zhao L D 2012 Energy Environ. Sci. 5, 8543.

[48] Charles Kittel 1986, Introduction to Solid State Physics, John Wiley \& Sons, Inc. New York.

[49] Sootsman J R, Chung D Y, and Kanatzidis M G 2009 Angew. Chem. Int. Ed. 48 8616-8639.

[50] Clarke D R 2003 Surf. Coat. Technol. 16367.

[51] Clarke D R, Levi C G 2003 Annu. Rev. Mater. Res. 33383.

[52] Cahill D G, Watson S K, Pohl R O 1992 Phys Rev B 466131.

[53] Zhao L D, Lo S H, Zhang Y, Sun H, Tan G, Uher C, Wolverton C, Dravid V P and Kanatzidis M G 2014 Nature 508373.

[54] Feng J et al. 2011 Acta Materialia 5917421760.

[55] Dresselhaus M S 2001 Solid State Physics Part I, Transport Properties of Solids.

[56] Reif 1965 Fundamentals of Statistical and Thermal Physics, McGraw-Hill pp. 393-397. 
[57] Rowe D M 2006 In Thermoelecrics handbook: macro to nano, edited by D. M. Rowe (Taylor and Francis, Boca Raton), pp. 1-1 thru 1-9. 